
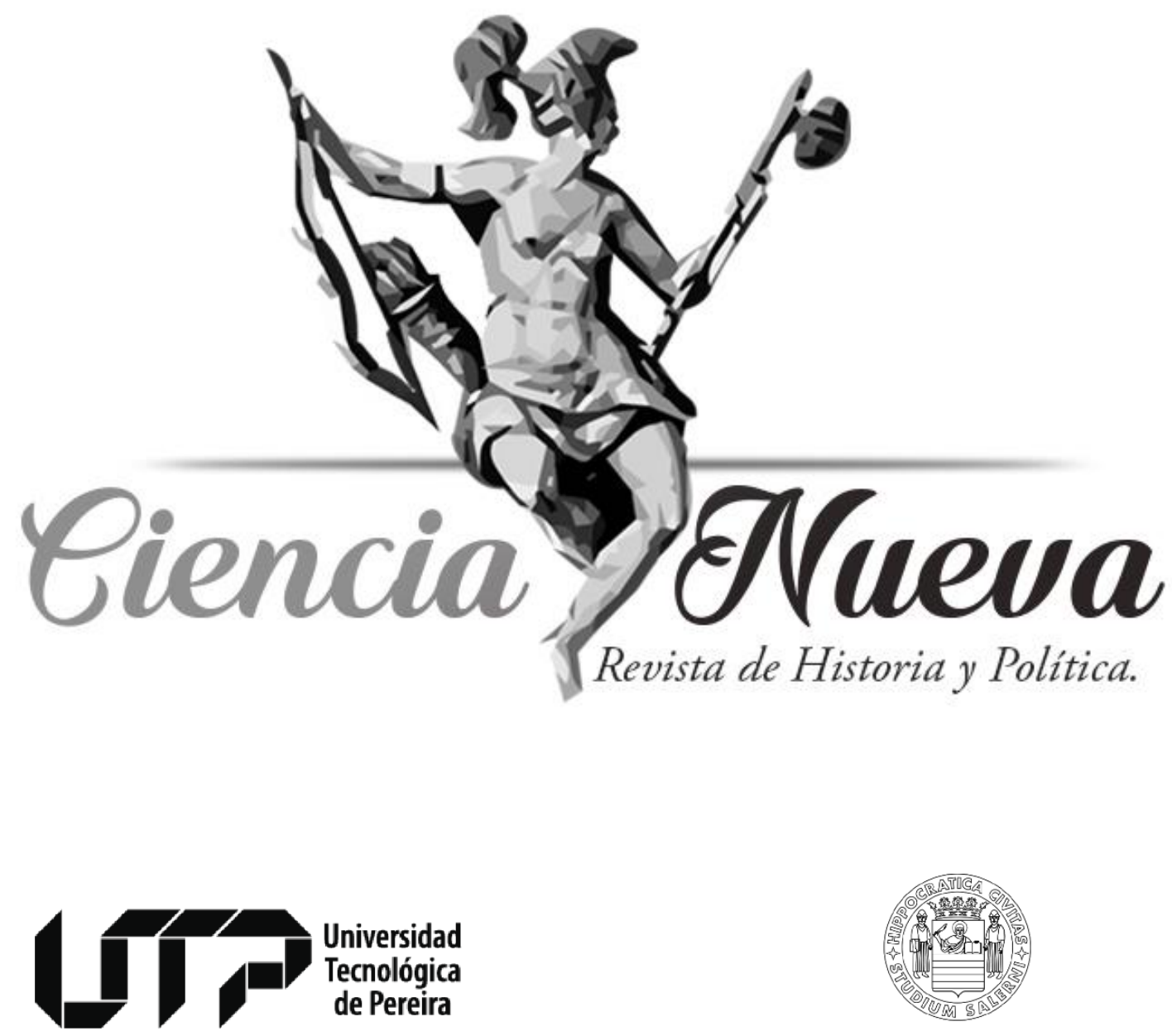

UNIVERSITÀ DEGLI STUDI DI SALERNO

Maestría en Historia

Maestría en Ciencia Política

RESEÑAS

ALONSO VALENCIA LLANO, AFRODESCENDIENTES EN EL VALLE DEL CAUCA: ENSAYOS HISTÓRICOS (CALI: PROGRAMA EDITORIAL UNIVERSIDAD DEL VALLE, 2016), 96 PP. ALONSO VALENCIA LLANO, AFRO-DESCENDANTS IN VALLE DEL CAUCA. HISTORICAL ESSAYS (CALI: PROGRAMA EDITORIAL UNIVERSIDAD DEL VALLE, 2016), 96 PP.

Paola Andrea Marín Velásquez pp. 168-172

Vol. $1 N^{o}$ 2, Julio-Diciembre de 2017 Pereira, Colombia 


\section{ALONSO VALENCIA LLANO, AFRODESCENDIENTES EN EL VALLE DEL CAUCA: ENSAYOS HISTÓRICOS (CALI: PROGRAMA EDITORIAL UNIVERSIDAD DEL VALLE, 2016), 96 PP." ALONSO VALENCIA LLANO,AFRO-DESCENDANTS IN VALLE DEL CAUCA. HISTORICAL ESSAYS (CALI: PROGRAMA EDITORIAL UNIVERSIDAD DEL VALLE, 2016), 96 PP.}

Paola Andrea Marín Velásquez.*

paola.marin@utp.edu.co

ORCID: http://orcid.org/0000-0002-1312-4761

\begin{aligned} & \hline Recibido: 06 de abril de 2017 \\ & Revisado: 30 de abril de 2017 \\ & Aceptado: 09 de junio de 2017 \\ & Publicado: 22 de agosto de 2017 \\ & \hline\end{aligned}

\section{A} lonso Valencia Llano es Licenciado en Historia de la Universidad del Valle, Magíster en Historia con mención en Historia Andina de la Facultad Latinoamericana de Ciencias Sociales (FLACSO) en Ecuador y Doctor en Historia de América de la Universidad de Pablo Olvide en Sevilla, España. Sus principales intereses investigativos se enmarcan en la historia política y los procesos de resistencia en el suroccidente colombiano, bandidos y políticas en el Valle del Cauca; al igual que la democracia, la guerra y la violencia en Colombia.

En su texto Afrodescendientes en el Valle del Cauca: ensayos históricos, el autor realiza una recopilación de varios artículos en relación al papel que han cumplido los afrodescendientes en el valle del río Cauca, en cuanto a sus procesos de resistencia y organización desde finales del periodo colonial en la Nueva Granada, hasta los primeros cincuenta años de la República, (siglos XVII-XIX).

El texto se encuentra estructurado en cinco capítulos: el primer capítulo, Aportes de los afrodescendientes al desarrollo del Valle del Cauca, describe cómo fue el proceso de poblamiento protagonizado por los campesinos negros, el intento de control de los mismos por parte de los criollos y su posterior papel en la lucha independentista. El segundo capítulo estudia la situación de los sectores populares en la independencia del Valle del Cauca, haciendo una relación de cómo ha sido narrada la "historia patria" con respecto a los campesinos y sus formas de economía, los pobres, la guerra, la posterior derrota de los españoles y la retaliación de los campesinos. El tercer capítulo, Los esclavos durante los primeros cincuenta años de las repúblicas andinas, explica de un modo detallado en qué consistió el sistema de esclavitud y las postrimerías del régimen colonial. Acto seguido, en el cuarto capítulo llamado Los esclavos y el proceso de independencia andina, Valencia expone su versión sobre la actitud de los esclavos frente a la independencia, la guerra de razas, la libertad de vientres y el Ejército patriota como medio de libertad. Por último, el autor

\footnotetext{
* El presente artículo respeta las directrices y normas dispuestas en la Declaración de Ética de Publicación de Ciencia Nueva, Revista de Historia y Política. Esta declaración puede consultarse en la página web de la revista: revistas.utp.edu.co/index.php/historia

** Estudiante de Licenciatura en Etnoeducación y Desarrollo Comunitario de la Universidad Tecnológica de Pereira, y de Trabajo Social en la Universidad del Valle Sede Zarzal.
} 
cierra con un capítulo donde, a modo de síntesis, relata el proceso de abolición de la esclavitud desde una perspectiva de larga duración en los nuevos Estados republicanos.

A partir de la compilación historiográfica sobre los afrodescendientes en el valle del río Cauca, resulta importante mencionar que el autor reconoce la capacidad que tuvieron para convertirse en "agentes de sus propios procesos históricos". Sin duda cumplieron un papel protagónico durante el periodo estudiado, lo que comúnmente no se reconoce en el historicismo clásico, el cual suele dar trascendencia solo a aquellas figuras representativas de los espacios sociales, políticos y económicos poderosos, invisibilizando a los afrodescendientes, actores significativos en la independencia y posterior construcción de la República.

A continuación, me propongo describir las características principales de cada uno de los artículos y ensayos históricos realizados por el historiador Alonso Valencia. En un primer momento, el autor explica que el aporte que han tenido los afrodescendientes en el valle del río Cauca no solo se han quedado en aspectos culturales, presentes en la gastronomía, las expresiones musicales y la tradición oral, entre otros, sino que también los afrodescendientes han contribuido al desarrollo económico del actual departamento del Valle del Cauca. En efecto, cabe recordar que en el momento en que la mano de obra de los indígenas fue diezmada considerablemente por el exterminio -sufrido especialmente durante el periodo colonial por parte de los invasores españoles en el siglo XVII-, como forma de sustitución, los esclavos negros provenientes de diversas regiones de África fueron introducidos no solo en la producción minera, sino que también trabajaron en las grandes haciendas dedicadas mayormente al cultivo de caña, tabaco, guarapo y mieles. Lo anterior significó un dinamismo en la economía de esa región, ya que respondía a la demanda de esos nuevos mercados.

Más adelante, esta situación generó un acelerado mestizaje. De manera que en el siglo XVIII, cerca del $60 \%$ de la población eran "libres de todos los colores". Lo que permitió el poblamiento disperso en zonas inundables y marginales, en donde los campesinos negros en el desarrollo de su producción económica, empezaron a competir con los grandes hacendados. Todo lo anterior como resultado de los procesos de resistencia y liberación de muchos esclavos; así como también de los consensos logrados con los criollos, debido a la crisis del declive de la minería en la región, sufrida en la primera mitad de siglo XVIII.

Por otro lado, el autor en el segundo capítulo, Los sectores populares en la independencia del Valle del Cauca, se encarga de cuestionar la tradición historiográfica del proceso de independencia, protagonizada por las élites criollas, dejando de lado los sectores populares representados en este caso por los afrodescendientes y los campesinos. De ese modo, menciona que la tradición de lucha de los sectores populares en contra de las élites locales y regionales, les dio "experiencia de negociación” con el Estado. Allí también resalta cómo la historia nacional toma un papel centralista, ignorando el papel histórico que las élites regionales tuvieron en la construcción de la República.

En el tercer capítulo, Los esclavos durante los primeros cincuenta años de las repúblicas andinas, el autor presenta la abolición de la esclavitud como una consecuencia de la actitud que tuvieron los esclavos en las postrimerías de la Colonia. Actitud caracterizada por las resistencias, las huidas al campo por parte de los cimarrones, el establecimiento de palenques y la búsqueda de autonomía. No obstante, aclara que no todos los esclavos hicieron resistencia, pues algunos buscaban formas legales de liberación. Lo que llama Valencia "estado de resignación", el cual se debía a que los esclavos ante la adquisición de mejoras económicas podían comprar su libertad, sometiéndose así a unas relaciones de dependencia. Allí el autor, apoyado en las ideas de William F. Sharp, demuestra que en el caso del Chocó, 
a pesar de que la esclavitud era útil en la Colonia, hubo un momento en el que fue necesario reducir la mano de obra negra. Esto debido a varias causas, entre las que se pueden destacar dos: el envejecimiento natural de los esclavos y lo rentable que resultaba la manumisión.

En el cuarto capítulo, Los esclavos y el proceso de independencia andina, se expone cómo durante el siglo XVIII el sistema esclavista de negros entra en crisis. A pesar de que durante un tiempo la esclavitud sufragó el problema de falta mano de obra, más tarde debido a la caída de la producción aurífera se hizo muy oneroso para los criollos manutener a sus esclavos. De otro lado, los negros se mostraban incrédulos ante las propuestas de "libertad" que les ofrecían para que entraran en los ejércitos independentistas, en parte, porque en muchas ocasiones, las promesas eran incumplidas y seguían siendo esclavos. En otros casos los utilizaban como "carne de cañón", acrecentando su desconfianza ante los criollos, lo cual ocasionó una "guerra de razas" (1810-1824) en contra de los "ricos y blancos" en las localidades, conflicto que favoreció a los españoles en detrimento de los patriotas.

En el último capítulo, el autor realiza unas conclusiones sobre el proceso de la abolición de la esclavitud. Si bien es cierto que este proceso fue lento por las distintas disputas e intereses, según Valencia, muchos de los afrodescendientes en la República continuaban en la esclavitud, puesto que las medidas se tomaron de manera generacional, es decir, las nuevas descendencias de negros fueron libres a través de la ley de vientres. También es fundamental aclarar que esta medida fue llevada a cabo de forma distinta en cada una de las regiones, porque la presencia de la esclavitud no tuvo la misma importancia económica y social, debido en parte a la prohibición del tráfico negrero por el parlamento británico, al igual que el incipiente desarrollo del sistema capitalista durante el siglo XIX.

Así pues, Valencia Llano aclara en su introducción que esta recopilación contiene artículos y ensayos históricos sobre los "afrodescendientes" en el Valle del Cauca, lo que significa que él no pretende abarcar todos los elementos sociales, económicos y políticos del periodo estudiado, sino que más bien procura analizar algunas situaciones específicas en las cuales los afrodescendientes tienen protagonismo. Por tanto, este estilo permite resaltar y dar énfasis a ciertas representaciones que demuestran el papel de la resistencia de estos grupos en la historia del sur occidente colombiano.

A primera vista, resulta importante cuestionar por qué el autor utiliza el concepto de "afrodescendiente" y habla del "Valle del Cauca" en el título de esta publicación, ya que el título no corresponde con el contenido del mismo. En otras palabras, afrodescendientes ${ }^{1}$ y Valle del Cauca son términos anacrónicos al momento histórico al cual se inscriben estos artículos, puesto que no corresponden al lenguaje usado en los siglos XVIII y XIX. Por un lado, para los "afros" la palabra habitual del momento era esclavos y negros; y por el otro, la zona analizada era la provincia del Cauca y no el departamento del Valle del Cauca, pues este solo se consolida a partir de la reforma político administrativa durante el mandato de Rafael Reyes en 1910.

Ahora bien, ¿Qué implicaciones puede tener el uso de un lenguaje anacrónico en la historia? bajo esta perspectiva tomo a Acosta que parafraseando a Renán Silva dice:

\footnotetext{
${ }^{1}$ El término afrodescendiente, políticamente, se acuña con mayor fuerza en el 2000 mediante la conferencia de Durban, en la cual según Rodríguez (2004): "Entramos negros y salimos afrodescendientes". Jorge Rodríguez Romero, "Entramos Negros; salimos Afrodescendientes", Revista Futuros 2, N 5 (2004). De esta manera se da una ruptura epistemológica en la evolución del concepto de negro, esto permite "reconocerse a sí misma como una comunidad étnica que politiza su identidad en tanto se considera un pueblo". Jhon Antón et al., Afrodescendientes en América Latina y el Caribe: del reconocimiento estadístico a la realización de derechos (Santiago de Chile: ONU, 2009), 20.
} 
El uso del lenguaje y de los conceptos marca definitivamente la calidad del análisis, su coherencia, su fortaleza interna en la comprensión y en el estudio de una sociedad. Llevar términos y categorías de nuestro tiempo para explicar fenómenos sociales acontecidos años o siglos atrás es un grave error que puede cambiar todo el análisis. Esta es la marca del anacronismo de la que los historiadores procuramos librarnos. Sin embargo, este error es mucho más sutil y delicado... ya que hay relaciones entre lenguaje y sociedad que debemos reconocer. También es interesante notar que, en las especialidades de los estudios del tiempo presente, los postcoloniales y subalternos está presente el problema del anacronismo cuando se utilizan las realidades del presente para explicar fenómenos del pasado...Lo propio sucede con los estudios postcoloniales cuando se llevan a cabo para sociedades y realidades ajenas a ese origen y contexto, con lo que se desnaturaliza la sociedad objeto de estudio. En esa medida, Renán Silva llama la atención sobre el lenguaje como "una de las primeras superficies con que nos encontramos cuando queremos conocer una sociedad". ${ }^{2}$ El llamado es a realizar una reflexión sobre el lenguaje como parte fundamental del trabajo del historiador; tener presente que las palabras, conceptos y categorías son susceptibles de historizar y pueden desorientar el análisis si no se es consciente de esa característica. ${ }^{3}$

Para terminar, cabe resaltar que el texto no solo está situado en el "Valle del Cauca", pues también da ejemplos de otras regiones mineras del occidente y norte de la Nueva Granada. Asimismo, menciona el proceso en otras latitudes de la zona andina como fue el caso de la Audiencia de Quito y el Virreinato del Perú, en los cuales a pesar de que hubo características similares de esclavitud, su abolición fue diferente.

\footnotetext{
${ }^{2}$ Renán Silva, Lugar de dudas. Sobre la práctica del análisis histórico. Breviario de inseguridades (Bogotá: Universidad de los Andes, 2015), 106.

${ }^{3}$ Yirla Marisol Acosta Franco, reseña de "Renán Silva, Lugar de dudas. Sobre la práctica del análisis histórico. Breviario de inseguridades (Bogotá: Universidad de los Andes, 2015), 228". Trashumante Revista Americana de Historia social 8 (2016):342-345.
} 


\section{Bibliografía.}

Acosta Franco, Yirla Marisol. Reseña de "Renán Silva, Lugar de dudas. Sobre la práctica del análisis histórico. Breviario de inseguridades (Bogotá: Universidad de los Andes, 2015), 228”. Trashumante Revista Americana de Historia social 8 (2016):342-345.

Anton, Jhon, Álvaro Bello, Fabiana Del Popolo, Marcelo Paião y Marta Rangel. Afrodescendientes en América Latina y el Caribe: del reconocimiento estadístico a la realización de derechos. Santiago de Chile: ONU, 2009.

Rodríguez Romero, Jorge. "Entramos Negros; salimos Afrodescendientes". Revista Futuros 2, $\mathrm{N}^{\circ} 5$ (2004).

Silva, Renán. Lugar de dudas. Sobre la práctica del análisis histórico. Breviario de inseguridades. Bogotá: Universidad de los Andes, 2015. 